\title{
Development of a Driver Model in Powered Wheelchair Operation
}

\author{
Takuma Ito $^{1}$, Takenobu Inoue ${ }^{2}$, Motoki Shino ${ }^{1}$, and Minoru Kamata ${ }^{1}$ \\ ${ }^{1}$ The University of Tokyo, Japan \\ ito@sl.t.u-tokyo.ac.jp \\ ${ }^{2}$ National Rehabilitation Center for Persons with Disabilities, Japan
}

\begin{abstract}
This paper describes the development of a driver model in a powered wheelchair operation. Existing methods have known problems such as straining the user. This is because improving wheelchairs adjustment requires too many trails and errors. Thus, we proposed solutions using computer simulation in this study. Computer simulation for the improvement of wheelchair adjustment needs three models: surroundings, driver and vehicle. Surroundings and vehicle models based on existing researches can be made, but not driver models for the computer simulation. To construct the model, we extracted the operation characteristics using a powered wheelchair simulator. From these results, we constructed the driver model as the first order preview driver model. In addition, a computer simulation was proposed for adjusting a powered wheelchair.
\end{abstract}

Keywords: Driver model, Powered wheelchair, Simulator, Operation characteristics.

\section{Introduction}

The number of persons with severe disabilities has been increasing in these days. It is necessary to offer mobility devices to them for social participation and the improvement of Quality of Life. A powered wheelchair is one of the effective devices. For the first step, some comfortlessness is allowed if the user can move in their own will. However, after the first step, comfort in moving is desired.

There are three elements to be evaluated for improving a powered wheelchair: surroundings, drivers and vehicles. Since these elements influence each other, the user is burdened from many trials and errors to improve his powered wheelchairs. Thus, we propose computer simulation solutions for this problem. The powered wheelchair driving computer simulation which contains the three elements makes it possible to adjust the powered wheelchair without lots of trials and errors.

\section{Research Question}

The powered wheelchair driving simulations need three models: surroundings models, driver models and vehicle models. Existing researches allow us to make surroundings 
models and vehicle models, but not a driver model. So, the purpose of this research is the development of a driver model in a powered wheelchair operation.

Driver models are originally proposed in the research field of automobiles ${ }^{[1]}$. They are mathematical expressions of vehicle operations, and are expressed by a transfer function, logical forms such as if-then rules and so on. Since powered wheelchairs and automobiles have many common characteristics, the concept of driver models for automobiles could be adjusted to powered wheelchair operation. However, a driver model for powered wheelchairs would be different from the driver models for automobiles, since powered wheelchairs and automobiles have also different characteristics.

\section{Experiment for Verifying the Possibility of Modeling}

\subsection{Hypothesis of This Experiment}

To model a powered wheelchair operation, we thought that following characteristics must be verified.

- The reproducibility of operation behaviors in the same driving condition

- The variance of operation behaviors under different driving condition

For these verifications, operation behaviors had to be measured quantitatively. However, since this measurement was realistically difficult, vehicle trajectory was measured instead of operations.

\subsection{Experiment Condition}

The subject was a 42 year-old male with C5 level paresis (from now on, he will be called Subject A). He had been using a powered wheelchair for 18 years since a spinal cord injury. He could use his left hand to operate the joystick of his powered wheelchair. He had no disability in the perception system.

An experimental course which imitated a corridor was made for this experiment. In this course, the subject was asked to conduct right turns and left turns in his usual way. As the experimental conditions, the width of the experiment course was changed by 5 steps: $0.9 \mathrm{~m}, 0.95 \mathrm{~m}, 1.0 \mathrm{~m}, 1.1 \mathrm{~m}, 1.2 \mathrm{~m}$. In each condition, 3 trials were tested. Measured data are the following:

- Vehicle position measured by motion capture (using a VICON).

- Movie of the operation recorded by 4 small cameras.

Fig.1 shows the appearance of the experiment.

\subsection{Experiment Result}

Fig. 2 shows right turn trajectories of the trials in course width $1.2 \mathrm{~m}$. From this figure, we confirmed the similarity of the trajectory under the same conditions.

In other conditions, this tendency was also confirmed. Thus, these results verify characteristics (a). (This is mentioned in the section 3.1)

Fig. 3 shows the average velocities in the right turns of each condition. Fig. 4 shows the average curvature radiuses in the right turns of each condition. From these figures, 


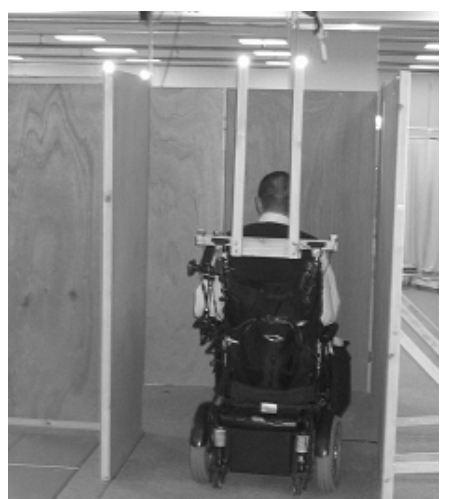

Fig. 1. Appearance of the experiment

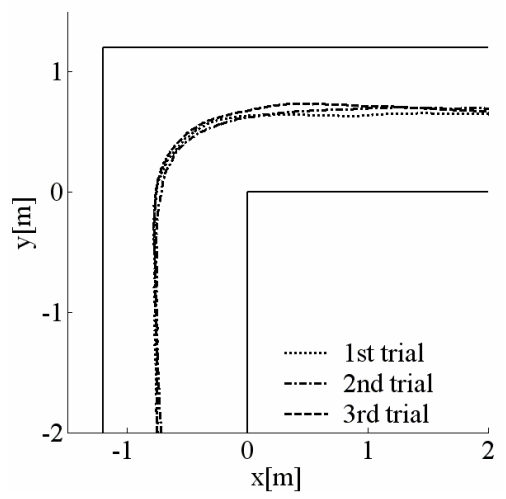

Fig. 2. Trajectory in course width $1.2 \mathrm{~m}$ (These lines are the trajectories of the center position between rear wheels)

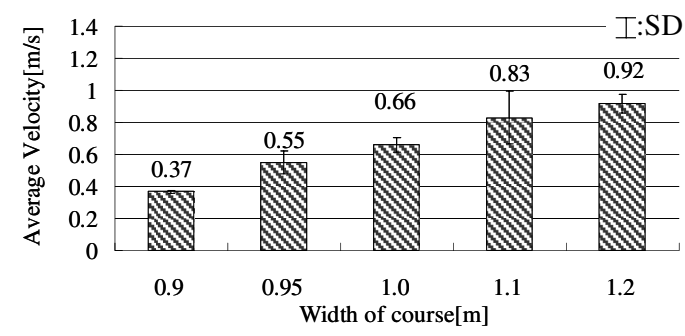

Fig. 3. Average velocity in width of each course

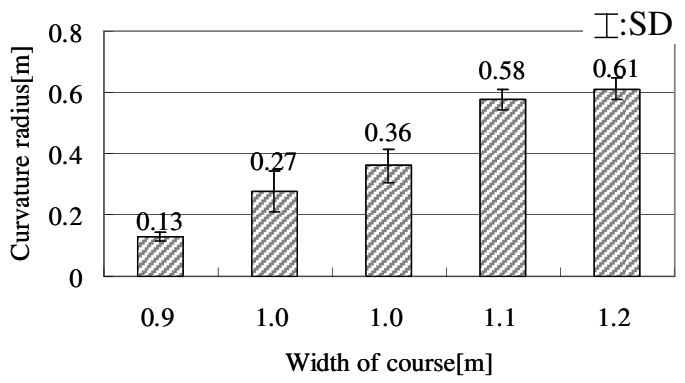

Fig. 4. Average curvature radius in width of each course

the response of the driver to the width of the experiment course was confirmed. Thus, these results verify characteristics (b). (This is mentioned in the section 3.1)

Changes of gaze points with facial motion were confirmed according to the phase of right turn by analyzing the operation movie. It seems that this result also indicates the driver's response to the driving environment.

The above-mentioned features were also confirmed in left turns. Therefore, we considered left turns and right turns to be equivalent. Henceforth, we determined right turns as the target operation. 


\subsection{Summary of This Chapter}

From the experiment results, the two operation characteristics mentioned in section 3.1 were confirmed. These characteristics verified the possibility of modeling powered wheelchair operation, and indicated model structures which needed external information inputs.

\section{Experiment for the Extraction of Driver Operation Characteristics}

\subsection{Before This Experiment}

To extract driver operation characteristics in detail, we tried a simulator experiment. Since many experiments were hard for the disabled subject, a subject with no disabilities (from now on, he will be called Subject B) was examined for this experiment. His validity as a subject was confirmed by a preliminary examination.

\subsection{Equipment of This Experiment}

Fig. 5 shows the appearance of the simulator. The simulator has 4 screens: two front screens which are projected from the rear side, and the other screens are projected from the front side by super-short focus type projectors. The horizontal viewing angles are 110 degrees and the vertical viewing angles are 55 degrees. At the bottom of the simulator, there is a 6-axes motion base. This motion base makes operators feel the acceleration of the vehicle. The input device is a joystick. For the calculation of vehicle motion, 3-dimentional dynamics model of a powered wheelchair ${ }^{[2]}$ was installed into the simulator system.

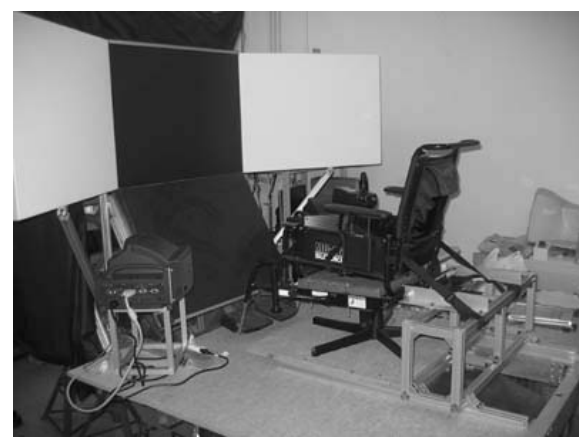

Fig. 5. Powered wheelchair simulator

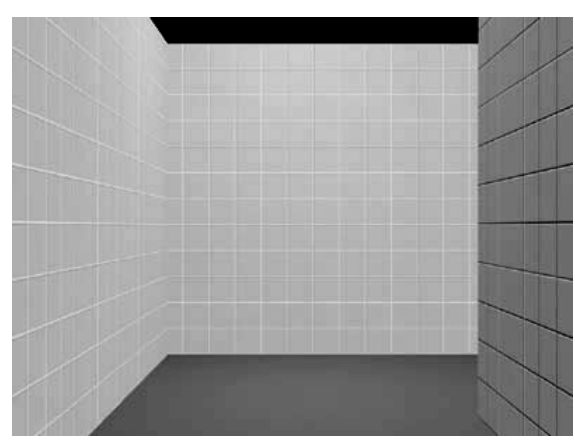

Fig. 6. Image of experimental course

\subsection{Experiment Condition}

Fig. 6 shows the appearance of the experiment course. The subject was given the following three instructions. 
- Drive through the corner.

- Don't hit the walls.

- Don't stop in the corner.

Acceleration and deceleration were allowed freely.

As the experimental conditions, the width of the experiment course and the vehicle maximum velocity were changed. The width was changed by 6 steps: $0.9 \mathrm{~m}, 1.0 \mathrm{~m}$, $1.2 \mathrm{~m}, 1.8 \mathrm{~m}, 2.0 \mathrm{~m}$ and $2.4 \mathrm{~m}$. The maximum velocity was changed by 9 steps: from $1.2 \mathrm{~km} / \mathrm{h}$ to $6.0 \mathrm{~km} / \mathrm{h}$ by $0.6 \mathrm{~km} / \mathrm{h}$. About 40 types of conditions were experimented combining the two conditions. To consider the reproducibility, more than ten trials on each condition were examined.

\subsection{Experiment Result}

As the characteristics of the operation, we analyzed the direction of the joystick. Fig.7 shows one typical example of the operation. 0sec means that the vehicle is $3 \mathrm{~m}$ short from the corner. $\theta_{\mathrm{js}}$ means the direction of the joystick and 0deg means the forward direction. From the analysis of the operation, the turning operation is divided into 3 phases. Phase 1 is the period before turning. In phase 1, the operation is around 0deg. Phase 2 is the period while turning. In phase 2, the operation almost constantly depends on the vehicle velocity. Phase 3 is the period after the turning. In phase 3 , the operation changes according to the distance to the walls. The purpose of the operation of this phase is to stabilize the powered wheelchair.

The operation changed according to the changes in the experimental conditions. The correction operation in phase 3 decreased when the maximum velocity was low or when the width of the course was wide. Inversely, when the maximum velocity was high and the width of course was narrow, the correction operation in phase 3 increased, and sometimes the correction operation even happened in phase 2.

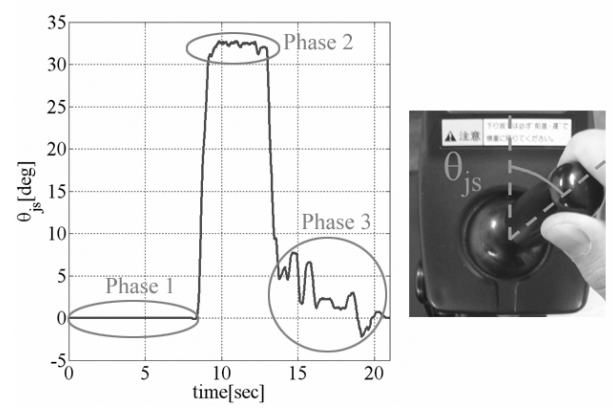

Fig. 7. Operation of $\theta_{\mathrm{js}}$ when turning to the right (subject B)

The reproducibility under the same condition was confirmed. Although the operation and trajectory both changed during two or three trials that preceded the changes in experimental conditions, the operation and trajectory were generally the same in each trial. However, in the extreme conditions when the maximum velocity is high and the width of course is narrow, the reproducibility is not confirmed. 


\section{Confirmation Experiment by a User with Disabilities}

\subsection{About This Experiment}

To confirm the generality of the previous experiment operation characteristics, a disabled subject was experimented. He was Subject A mentioned in chapter 3.

Because Subject A had disabilities and needed to be reclined and steadily fixed to the chair, the experiment was conducted with the powered wheelchair simulator of National Rehabilitation Center for Persons with Disabilities. The screens and seat were a little different from the previous simulator, but the input device and the calculation system were same.

In this experiment, the number of experiment conditions was reduced concerning the load of the subject. The width of the course was fixed to $1.2 \mathrm{~m}$. Two kinds of conditions were prepared for the velocity settings. One was the maximum velocity setting. In this kind of conditions, the subject could adjust the velocity freely. The maximum velocities were $2.4 \mathrm{~km} / \mathrm{h}$ and $3.6 \mathrm{~km} / \mathrm{h}$. The other was the constant velocity setting. In this kind of conditions, the subject could not adjust the velocity. The constant velocities were from $1.2 \mathrm{~km} / \mathrm{h}$ to $3.0 \mathrm{~km} / \mathrm{h}$ by $0.6 \mathrm{~km} / \mathrm{h}$. In each condition, 10 trials were experimented after a few practice trials.

\subsection{Experiment Result}

Fig. 8 shows one typical example of the operation. From this figure, the following operation characteristics were confirmed in common.

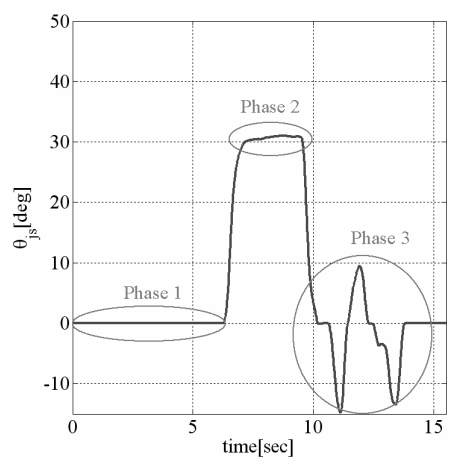

Fig. 8. Operation of $\theta_{\mathrm{js}}$ when turning to the right (subject A)

- The 3 phases in operation segmentation

- The operation characteristics in each phase

The reproducibility of operation behaviors in the same driving condition and the response of operation behaviors to the change in the driving conditions were also confirmed. These results were common characteristics to the subject B. Thus, it seems that a driver model in a powered wheelchair operation could be developed from these common characteristics. 


\section{Construction of a Driver Model}

\subsection{Mathematical Expression}

We constructed a driver model in a powered wheelchair operation. The expression of the driver model is shown in the following.

$$
\begin{array}{ll}
\theta_{j s}=\frac{1}{1+\tau_{\mathrm{d}} s} \mathbf{h} \cdot \boldsymbol{\Delta} & \left(\left|\theta_{j s}\right| \leq \theta_{m}\right) \\
\theta_{m}=\mathrm{k} \cdot u+\mathrm{c} & : \text { Reaction time } \\
\tau_{\mathrm{d}} & : \text { Laplace operator } \\
s & : \text { Operation gain vector (2 elements) } \\
\mathbf{h} \equiv\left(\mathrm{h}_{\mathrm{i}}-\mathrm{h}_{\mathrm{o}}\right) & : \text { Preview distance vector to the nearer wall (2 elements) } \\
\boldsymbol{\Delta} \equiv\left(\left(1+\tau_{\mathrm{p}} s\right) d_{i}\right) & : \text { Preview time } \\
\tau_{\mathrm{p}} & : \text { Operation limit } \\
\theta_{m} & : \text { Operation limit parameters } \\
\mathrm{k}, \mathrm{c} & : \text { Velocity } \\
u &
\end{array}
$$

This model is based on the first order preview driver model. The operation is determined depending on the preview distance vector to the nearer walls. Fig.9 shows the definition of the distance vector. $\tau_{\mathrm{p}}$ means the preview time of the driver operation. When a driver operates the vehicle, the driver uses the information not in the present position but in the future position. How far in the future the information that the driver uses is almost constant, so it is expressed as a constant time $\tau_{\mathrm{p}}$. $\mathbf{h}$ means the operation gain. Since drivers tend to margin inside in the corner, $\mathrm{h}_{o}$ is usually larger than $h_{i} . \tau_{d}$ means the reaction time of the driver operation and it is the neuromuscular delay of the driver. $\theta_{m}$ means the operation limit. Too large yawing motion is uncomfortable for the driver. Even if the calculated $\theta_{j s}$ is the appropriate value, drivers don't operate lager than $\theta_{m}$. The model parameters are calculated from the result of the previous experiments.

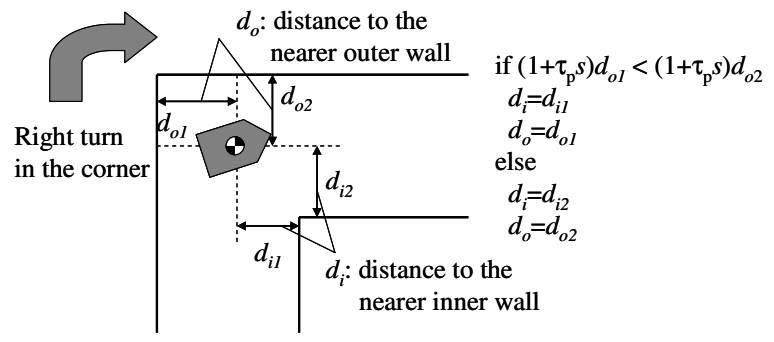

Fig. 9. Definition of $d_{i}$ and $d_{o}$ 


\subsection{Verification of the Driver Model}

To verify the driver model, a closed-loop computer simulation with the driver model was tested. Fig.10 shows the computer simulated operation in comparison with experiment result. Fig. 11 shows the computer simulated trajectories in comparison with the experiment result. From fig. 10, some similarities could be confirmed: operation amount and operation timing. From fig. 11, even though a little difference is confirmed in the corner, the two trajectories seem to be similar in general. From these results, we confirmed the validity of this driver model.

\section{Driver Model Application}

\subsection{Proposal of an Evaluation Simulation with a Driver Model}

As we pointed out in the chapter 1, the adjustments of powered wheelchairs need a lot of trials and errors. So we propose the evaluation computer simulation with a driver model. Optimization for the subject who is the prototype of the driver model could be possible by building the driver model into a closed-loop computer simulation.

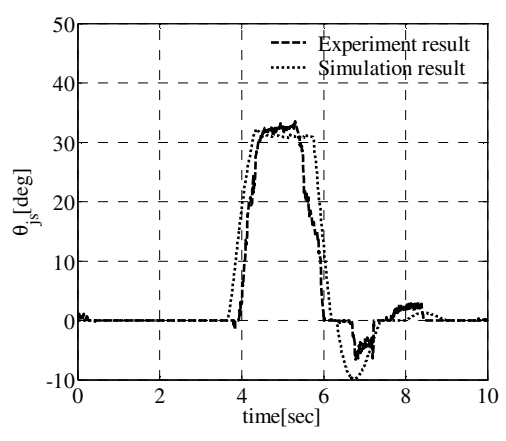

Fig. 10. Simulation result (Operation amount)

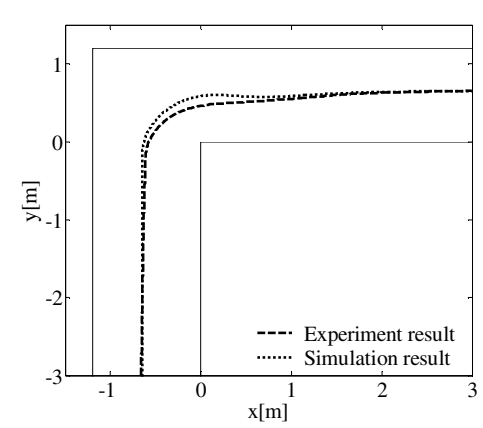

Fig. 11. Simulation result (trajectory)

To confirm the effectiveness of this idea, we tried the computer simulation for adjusting the maximum speed parameter of a powered wheelchair. The evaluation index was the integral value of the operation and the maximum speed parameter was ordered with this evaluation. On the other hand, the subjective evaluation of the maximum speed parameter was examined with the powered wheelchair simulator. Both the prototype of the driver model and the subject is subject B (mentioned in chapter 4).

\subsection{Evaluation Result}

Table 1 shows the results of the subjective evaluation and the computer simulation evaluation. Similar tendency could be confirmed between these results, even though a little difference is confirmed about parameter $3.6 \mathrm{~km} / \mathrm{h}$. This indicates the possibility that the computer simulation approach could substitute of an experiment adjustment. Thus, we think the effectiveness of this simulation is confirmed from this result. 
In this case, the evaluation index is the integral value of a computer simulated operation. This index means that the less operation is the better. However, other indexes could be effective. For example, integral value of the distance to the wall could evaluate the risk of driving, maximum yawing motion and acceleration would evaluate the comfort of a driver, and so on. About the selection of the evaluation index, there would be room to discuss.

\section{Discussion}

In this research, we constructed a driver model from a few experiment results. However, the number of the subjects was only two. Therefore, verification of the model was confirmed only in the subjects. To verify the driver model more clearly, more subjects and their experiment data are needed. This is a future task.

Table 1. Results of subjective evaluation and simulation evaluation

\begin{tabular}{|l|l|l|}
\hline $\begin{array}{l}\text { Max velocity } \\
\text { parameter(km/h) }\end{array}$ & $\begin{array}{l}\text { Subjective } \\
\text { Evaluation order }\end{array}$ & $\begin{array}{l}\text { Integral value of simulated } \\
\text { operation amount(deg.sec) }\end{array}$ \\
\hline 2.4 & 1st & 71.18 \\
\hline 3.0 & 2nd & 72.81 \\
\hline 1.8 & 3rd & 91.93 \\
\hline 1.2 & 4th & 181.71 \\
\hline 3.6 & 5th & 73.54 \\
\hline
\end{tabular}

(Note: The less integral value is, the better evaluation is.)

There are other future tasks. As a driver operation, the turning operation in corners was focused for the development of the driver model. However, not only turning but also acceleration is important in powered wheelchair operations. So, the proposed model lacks and needs acceleration operations in the modeling.

As environment conditions, the proposed driver model was constructed and tested in a course with a width of $1.2 \mathrm{~m}$. How drivers react to other environment conditions is important. So, the proposed model also lacks generalization to the environmental elements. In this point, environmental elements could be solved as a parameter of the driver model. However, since we can not state clearly about this, it is also a future task.

\section{Conclusions}

This paper developed a driver model of a powered wheelchair operation. The following findings were obtained by the process of this research.

1. A simulator of a powered wheelchair was developed for the analysis of operation.

2. The operation characteristics of a powered wheelchair were examined. 
3. A driver model of a powered wheelchair operation was constructed as the first order preview driver model.

4. The computer simulation for adjusting maximum speed parameter of a powered wheelchair was proposed.

\section{References}

[1] Plochl, M., et al.: Driver models in automobile dynamics application. Vehicle System Dynamics 45(7-8), 699-741

[2] Yamakawa, Y., et al.: Development of Electric Wheelchair with Operational Force Detecting Device for Persons with Severe Disability (in Japanese). In: Proceedings of the Welfare Engineering Symposium, vol. 2006, pp. 39-42 (2006) 